\title{
Earthquake response analysis of soil-rock slope based on distribution of rocks
}

\author{
Huang Xianwen, Liu Shunqing, Sui Xiaolan ,Hu Yuan. \\ Jiangsu University of Science an Technology,Zhen Jiang,212000,China
}

\begin{abstract}
Soil-rock slope is a structure of slopes distributing widely in the world, but the calculation model of its stability is always set as uniform soil model under the influence of earthquake, and the effect of rock distribution on the slope can't be considered comprehensively. Therefore, based on the method of finite element non-linear time-procedure, this paper analyses the acceleration response of slopes under the influence of earthquake with different rock distribution. Results show that the distribution of rocks have great influence on the accelerated response characteristic, and the main findings are as follows: (a) the accelerated time-history curve can filter the high frequency waves; (b) the free-surface has amplifying effect on seismic waves; (c) the acceleration response curves have different degrees of "biased". Through above analysis, it's expected to provide some reference for the stability analysis of soil-rock slope and reinforcement scheme under the influence of earthquake.
\end{abstract}

\section{Introduction}

Soil-rock slope is a kind of mixed-media slopes ${ }^{[1]}$, consisting of rocks that have high strength and density, and soil particles which have low strength and small particles, which distributed widely in China ${ }^{[2]}$. On 12th May, 2008, a 8.0-magnitude earthquake occurred in Wenchuan, which resulted in a large number of landslides, and caused heavy casualties and enormous property loss. According to surveys, massive landslides, caused by earthquakes, including Yushu earthquake, Yingjiang earthquake, and Jiuzhaigou earthquake, contain a lot of rocks and gravel. Especially in recent years, earthquakes happened frequently, triggering landslides and debris flow. As a kind of slopes, the soilrock slope has non-homogeneous material characteristics, which making its response characteristics under seismic loading different from the traditional uniform soil slope.

In recent years, many scholars inland and aboard have analyzed the stability of slopes under the effect of dynamic loads. Davis et al. ${ }^{[5]}$ monitored the San Fernando earthquake and found that the slope has an amplifier effect on seismic waves; then Zhang Zhuoyuan $^{[6]}$ and Hideo Takano ${ }^{[7]}$ analyzed the seismic monitoring results of slopes, and found that the amplifier effect of the slope under seismic loading is closely related to slopes' height and the constituent materials of the slope. The actual monitoring results can reflect the response dynamic characteristics of the slope under seismic loading. However, the differences of slopes, the specificity of the seismic waves, and many uncertainties make it difficult to analyze the dynamic response systematically.
Ren Ziming et al. ${ }^{[8]}$ used large-scale shaking table tests to analyze the stability of the slope under seismic loading. The results showed that the slope has obvious amplifier effect on the seismic waves, and the amplifier effect was the smallest at the slope shoulder, and with the increase of the slope gradient, the amplifier effect of acceleration and velocity weakened gradually. Liu Hanxiang et al. ${ }^{[9]}$ studied the effects of seismic wave types, excitation directions, and ground motion on the horizontal acceleration of the slope through a shaking table test. The results showed that the acceleration amplifier effect decreases with the increase of the elevation and strengthen with the increase of the vibration intensity. The slope shaking table test about scale model can more reliably reflect the dynamic response characteristic of the slope under seismic loading. However, The complicated preparatory work and instrument installation process limit the application of scale model tests in engineering.

With the development of computer technology, many analysis methods, considering dynamic loading, have been realized. Zheng Yingre ${ }^{[10]}$ et al. pointed out the shortcomings of the traditional pseudo-static method in dynamic stability analysis of the slope, and proposed a dynamic finite element time history analysis method and a finite element strength reduction dynamic analysis method based on the tension-shear failure. The finite element software FLAC3D was used to verify the correctness of the theory through establishing corresponding model. According to the actual situation of the Wenchuan earthquake, Men $\mathrm{Ni}^{[11]}$ et al. established an analytical model by using ANSYS software, to study the corresponding relation between 
resonant characteristics of the slope, characteristics of seismic response and earthquake ground motion. Chen Lingling ${ }^{[12]}$ et al. used the methods of time history analysis and response spectrum analysis to study the dynamic characteristics of the slope under seismic loading, and obtained the calculation formula for evaluating the stability of the rock slope. In the above analysis of the slope dynamic response characteristics, the slopes were all set as uniform soil slopes. However, in actual practice, slopes often contain rocks, forming soil-rock slopes. The difference in material properties and mechanical properties between rocks and soil makes the dynamic response characteristic of the slope have some differences. Therefore, it is necessary to study the dynamic response of soil-rock slope with large stone.

Based on the above reasons, according to the differences in distribution location of large rocks in soilrock slopes, this paper analyzes the accelerate response characteristics of slopes under seismic loading by using the nonlinear time history analysis function of MIDASGTS to guide the support engineering of soil-rock slopes .

\section{Calculation Principle}

\subsection{The calculation method of seismic loading}

Time-history analysis method ${ }^{[13,14,15]}$ is a common calculation method in slope dynamic calculation whose results have greater difference from the methods of traditional static calculation. On the one hand, timehistory analysis considers displacement, velocity and acceleration varying with time under dynamic loading; compared with the single solution in traditional static analysis, it has more solutions, so that it can better reflect the time effect of the structure under dynamic loading. On the other hand, the considerations of dynamic response in time-history analysis are more extensive, and the equations of displacement, velocity and acceleration need to establish under dynamic loading. It is necessary to consider the damping effect for non-elastic materials, making the solution more complicated.

The equilibrium equation under dynamic loading:

$$
[M]\{\ddot{u}\}+[C]\{\dot{u}\}+[K]\{u\}=\{F(t)\}
$$

which $[\mathrm{M}]$ is the total mass matrix; [C] is the total damping matrix; $[\mathrm{K}]$ is the total stiffness matrix; $\ddot{u}$ is acceleration vector; $\dot{u}$ is velocity vector; $\ddot{u}$ is displacement vector; $\{F(t)\}$ is the whole nodal force vector. When the seismic force is loaded on the structure, it is added into the dynamic balance equation in the form of nodal force. The corresponding formula is as follows:

$$
[M]\{\ddot{u}\}+[C]\{\dot{u}\}+[K]\{u\}=-[M][I] \dddot{u}_{e}(t)
$$

Which $\dddot{u}_{e}(t)$ is seismic acceleration force; [I] is identity matrix. In MIDAS-GTS, the equation is mainly solved by the Newmark method. By establishing the second-order relationship between time increment, $\Delta t$ velocity $\dot{u}$, and displacement $u$, a more accurate numerical solution can be obtained.

\subsection{Damping Matrix}

In the non-damping analysis system, the structure will be in infinite circulation movement after the external force disappearing, which is significantly different from the actual structural response, especially in the slope structure. Generally, when the action of the dynamic loading is over, the slope will gradually become static under the damping action, instead of cyclic motion. The reason is that the soil particles are disturbed by the action of dynamic loading such as earthquake, resulting in relative displacement and deformation, which will consume a certain amount of energy. Therefore, with the consumption of energy, the slope motion will also stop gradually. The complex mechanism and the causes of damping make it difficult to calculate with a single variable accurately. Rayleigh introduced the linear damping into elastomer vibration equations. After analyzing and simplifying, the viscous damping matrix, related to the structural stiffness and mass, was established:

$$
[C]=\alpha[M]+\beta[K]
$$

Where $\alpha, \beta$ denotes the mass and stiffness influence coefficient respectively whose matrix solves the problem of damping matrix calculation in structural dynamic analysis accurately.

\subsection{Boundary conditions}

Seismic waves generally start from the basement. When arriving at the slope surface, the transfer medium mutates and causes reflections. The reflected wave propagates around the soil and dissipates at infinity. However, it is impossible to set up the model with semiinfinite soil mass for the limitation of the calculation unit which highlights the importance of suitable boundary conditions. In the traditional static analysis, the slope bottom is a fixed end, and horizontal constraint is set on both sides. Under above assumptions, seismic waves don't dissipate at the boundary, but generate reflected waves again, which are continuously reflected within the slope model, producing a "box effect". It makes the dynamic response of the slope under seismic loading be falsehood, especially when the size of model is small, a large number of reflected waves make the results greatly different from the actual situation. Therefore, it is necessary to establish a spring-damper at boundary of the model to absorb the energy and balance the displacement to stop waves reflect. In MIDAS-GTS, the transfer boundary conditions can consider the consumption characteristics of reflection waves well.

\section{Finite element model}

\subsection{Geometric model}

The corresponding finite element model is built according to a soil-rock slope in Zhenjiang (Fig.1). The total width of the calculation model is taken as $100 \mathrm{~m}$; the soil height before the slope is $25 \mathrm{~m}$; the soil height after 
the slope is $50 \mathrm{~m}$; the slope height is $25 \mathrm{~m}$, and the slope width is $15 \mathrm{~m}$.

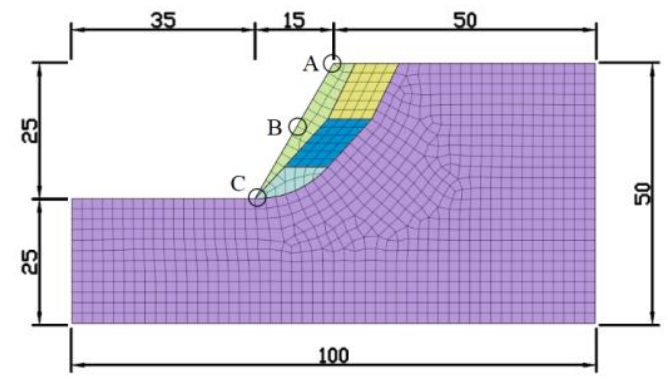

Fig. 1 Finite element calculation model

The corresponding boundary conditions are set up: firstly, the transfer boundary conditions are applied on the left and right sides of the slope, including viscous boundary and free-damping; then apply transfer boundary conditions to the bottom of the slope, meanwhile introduce horizontal and vertical fixed constraints to ensure the input of seismic loading.

\subsection{Material constitutive}

In static state, the elastic modulus of soil and rock is fixed. Under dynamic load, the elastic modulus of the material decreases due to the mutual movement between the particles, which manifesting as the gradually nonlinearity decay of dynamic elastic modulus. As the strain increasing, the energy consumption that the particles moving bring about more; the damping ratio is increasing gradually, presenting non-linear characteristics. In order to consider the dynamic response characteristics of the slope under dynamic loading accurately, the constitutive features of the material are simulated by using a two-dimensional equivalent linear model. The material physical parameters are shown in Table 1, and the stress-strain relationships are shown in Fig.2.

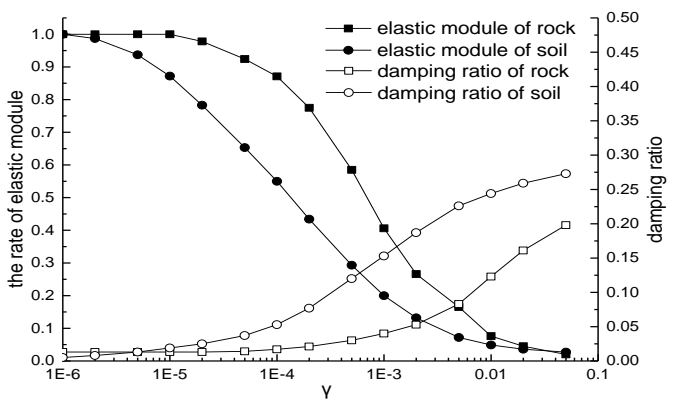

Figure 2 The strain compatible equation of the material

Table 1 Material physical parameters

\begin{tabular}{ccccc}
\hline $\begin{array}{r}\text { com } \\
\text { position }\end{array}$ & $\begin{array}{c}\text { den } \\
\text { sity } \\
\mathrm{kN} / \\
\mathrm{m}^{3}\end{array}$ & $\begin{array}{r}\text { shear } \\
\text { modulus } \\
\mathrm{kPa}\end{array}$ & $\begin{array}{c}\text { poi } \\
\text { sson } \\
\text { ratio }\end{array}$ & $\begin{array}{c}\text { damp } \\
\text { ing ratio }\end{array}$ \\
\hline soil & 1.8 & $1.2 \mathrm{e} 4$ & 0.3 & 0.05 \\
rocks & 2.5 & $1.2 \mathrm{e} 5$ & 0.2 & 0.05 \\
\hline
\end{tabular}

\subsection{Earthquake force input}

The EI-Centro seismic wave is the first seismic wave recorded in the world, and the complete cycle is 54s. In this paper, the representative top 20 s of the seismic wave is selected as the seismic acceleration to input. The calculation step is $0.01 \mathrm{~s}$ and he horizontal acceleration curve is shown in Fig.3.

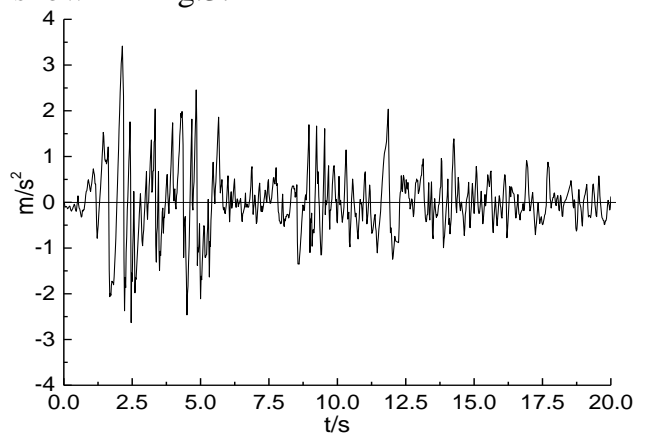

Fig. 3 Horizontal Acceleration Spectrum of EI-Centro Earthquake

\subsection{Analysis model}

The response characteristic of slopes under seismic waves differs from layered soil due to the existence of "free-surface". The large rocks with higher density make the response characteristic of earthquake different from the homogeneous soil, when there are large rocks distributed on the slope surface. Through analysis, it is found that the distributions of greater rocks have great influence on the response characteristic of slopes under seismic loading on the surface. Based on the difference of rocks, four distribution models are established: the height of the areas II, III, and IV is respectively taken as the trisection points of the plastic zone length of homogeneous soil slope, and the calculating width is taken as the width of the top surface of the uniform slippery body. The area I is the undivided area of the sliding mass, as shown in Fig. 4:

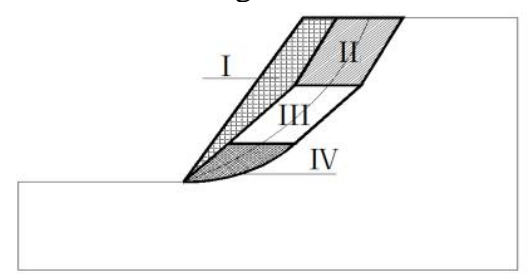

Figure 4 distributions locations of rocks

\section{Dynamic response analysis}

4.1 The acceleration response of homogeneous soil slope 


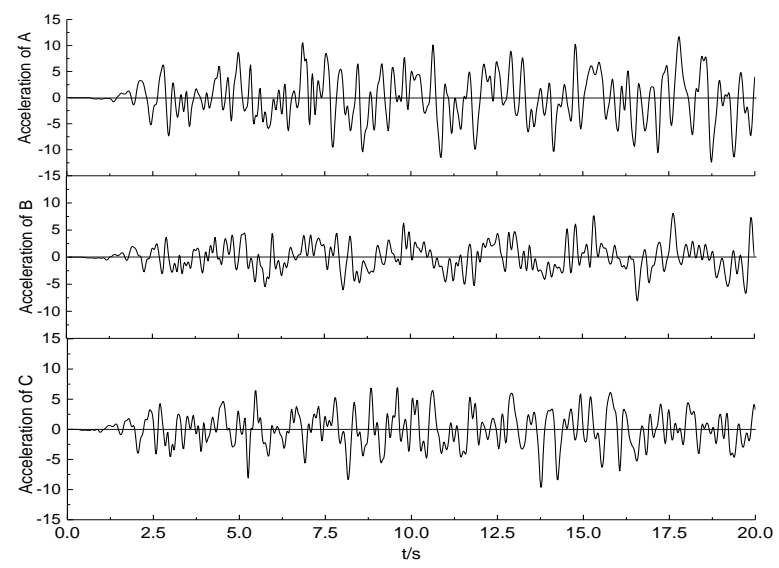

Figure 5 Acceleration response of homogeneous soil

As shown in Figure 5, the acceleration response curve at each point of the slope is significantly different from the curve of input earthquake acceleration.

Comparing with the acceleration time histories at toe of slope ( $\mathrm{C}$ point) with the input seismic wave, it is found that the acceleration change period is longer than the input earthquake acceleration, and the two above differ in the variation trend: the original seismic wave passes through the ascending stage of $2.14 \mathrm{~s}$, reaching the maximum acceleration of $3.42 \mathrm{~m} / \mathrm{s}^{2}$, then gradually decreases, and rise again, finally it gradually decay. The acceleration at toe of slope slowly increases after a rapid rise of $2.5 \mathrm{~s}$, reaching a peak acceleration of $9.6 \mathrm{~m} / \mathrm{s}^{2}$ at $13.76 \mathrm{~s}$, and then it gradually decay. For the damping effect of the cohesive soil, the soil at toe of slope showed skewed popularity in response to seismic acceleration, and has a certain reduction effect on the high-frequency part of the seismic wave, which is specifically expressed as the filter effect. There are more differences between the acceleration response of the mid-slope (point B) and the original seismic input curve. It has a filter effect, but the acceleration uniform changes with some bias on the side of the equilibrium position, arriving at the maximum acceleration $8.11 \mathrm{~m} / \mathrm{s}^{2}$ at $17.64 \mathrm{~s}$. The existence of free surface is the main reason for the deflection of mid-slope acceleration curve; the damping effect of cohesive soil makes the emergence time of the maximum peak to lag behind the acceleration time histories at toe of slope. The acceleration time histories curve differ and

The acceleration time-history curve of point $\mathrm{A}$ is different from that of point $\mathrm{B}$ and $\mathrm{C}$, which mainly reflects in the acceleration response amplitude. The maximum peak acceleration is $11.49 \mathrm{~m} / \mathrm{s}^{2}$ at $18.74 \mathrm{~s}$. Because of the influences of the slope top and the free surface outside the slope, the soil at the top of the slope is less constrained by the seismic force. As a result, there is a large acceleration response, and the amplification characteristics of the seismic wave are more obvious.

\subsection{The acceleration response of rocks in area I}

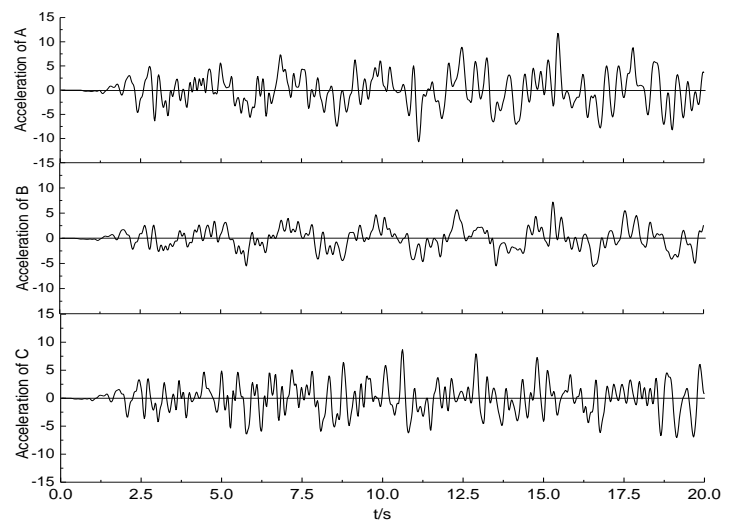

Figure 6 The acceleration response of rocks at area I

When there are more rocks on the slope (area I), the dynamic response of the slope is significantly different from that of the traditional homogeneous soil slope.

Comparing with the acceleration response curve at the toe of slope ( $\mathrm{C}$ point), it is found that the slope acceleration response curve of the slope with rocks is more regular. After rising phase of $2.5 \mathrm{~s}$, the acceleration amplitude basically stabilizes in a range and reaches the peak acceleration $8.66 \mathrm{~m} / \mathrm{s}^{2}$ at $10.62 \mathrm{~s}$. Sudden increasing is less likely to happen. When the rocks is distributed on the slope, the relative mass of the rocks makes it to have greater inertial force, which restricts the acceleration at toe of slope to a certain extent, and it makes the acceleration response at toe of slope showing the characteristics of homogenization .

Comparing with the acceleration response curve of point B, the "bias" of acceleration response is more obvious. Rocks with larger mass make it difficult for the mid-slope acceleration to change direction in a short period. Therefore, the acceleration response just changes on one side of the equilibrium position. After a rapid increase of $2.5 \mathrm{~s}$, the acceleration reaches a peak acceleration of $7.2 \mathrm{~m} / \mathrm{s}^{2}$ at $15.32 \mathrm{~s}$, lagging behind that of toe of slope significantly.

Comparing with the acceleration response curve at point $\mathrm{A}$, the acceleration change period is shorter and the filter effect is more obvious. On the one hand, the filter effect of cohesive soil is more obvious due to the larger quality of the rocks on the slope; On the other hand, the characteristics of the free-surface on the slope top and slope surface make the acceleration response on the slope top more obvious, it reaches a maximum at $15.46 \mathrm{~s}$, and the peak acceleration is $11.75 \mathrm{~m} / \mathrm{s}^{2}$.

\subsection{The acceleration response of rocks in area II}

When rocks is distributed on the slope top (area II), the difference between the acceleration response of toe of slope and the response pattern of the homogeneous soil slope is mainly reflected in the acceleration amplitude and the occurrence time period. The acceleration at point $\mathrm{C}$ steadily rises in the first $10 \mathrm{~s}$, and maintains a maximum acceleration at $10.78 \mathrm{~m} / \mathrm{s}^{2}$ for about $5 \mathrm{~s}$. Finally the acceleration decreases gradually. It is different that the homogeneous soil slope keeps the stability time for about $10 \mathrm{~s}$ after the rise time for about $5 \mathrm{~s}$. 
The acceleration time-history curve of point $\mathrm{B}$ is similar to the response characteristics of homogeneous soil slope, but varies in the magnitude value. The maximum acceleration at point $\mathrm{B}$ achieves $6.16 \mathrm{~m} / \mathrm{s}^{2}$ at 19.1s. It shows that the distribution of rocks on the slope top has certain reduction effect on the acceleration response amplitude at mid-slope.

When the larger rocks are distributed at point $\mathrm{A}$, the acceleration response curve at slope top is similar to that of the homogeneous soil slope, but the amplitude decreases. The maximum acceleration at point $B$ achieves $10.23 \mathrm{~m} / \mathrm{s}^{2}$ at $17.8 \mathrm{~s}$. It shows that the rocks on the slope top has a certain reduction effect on the acceleration response at slope top.

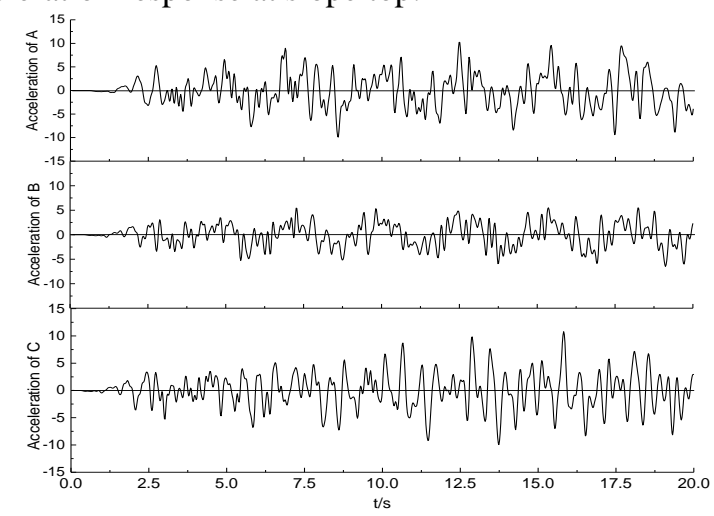

Figure 7 The acceleration response of rocks at area II

\subsection{The acceleration response of rocks in area III}

As shown in Figure 8, when rocks is distributed in the slope (area III), the maximum acceleration at point $\mathrm{C}$ achieves $10.47 \mathrm{~m} / \mathrm{s}^{2}$ at $17.96 \mathrm{~s}$, which is bigger than the maximum acceleration of the homogeneous soil slope. By comparing to the two images, it is found that the accelerated response of the rocks in the slope is more intense than that of the homogeneous soil slope; the peak acceleration is greater than that of the homogeneous soil slope, and the occurrence number is more. It demonstrates that the distribution of rocks in the slope has a certain amplification effect on the acceleration response at the toe of slope.

When rocks was distributed in the slope, the difference between the acceleration response of the midslope (point B) and the homogeneous soil slope is mainly reflected in the cycle of acceleration vibration. The presence of rocks in the slope makes the highfrequency acceleration response of the slope decreasing, and the overall response characteristics are similar to that of the homogeneous soil slope. The peak acceleration occurs at $16.52 \mathrm{~s}$ and the amplitude is $6.24 \mathrm{~m} / \mathrm{s}^{2}$, which is lower than that of the homogeneous soil slope. It shows that the rocks accumulate in the slope to enhance the "hysteresis" of the acceleration response of the slope, and has a certain reduction effect on the acceleration amplitude.

Compared with the homogeneous soil slope, the acceleration response at point $\mathrm{A}$ is mainly reflected in the "bias" of the acceleration curve and the amplitude of acceleration. The maximum acceleration of the slope occurs at $13.56 \mathrm{~s}$ and the size is $12.9 \mathrm{~m} / \mathrm{s}^{2}$. It shows that the rocks in the slope have a significant amplification effect on the acceleration at the slope top. The reason is that the large inertial force of the rocks in the slope changes the acceleration of the earthquake transmitting to the slope top, and the characteristics of the double free surface at the slope top make the acceleration response of the slope obvious.

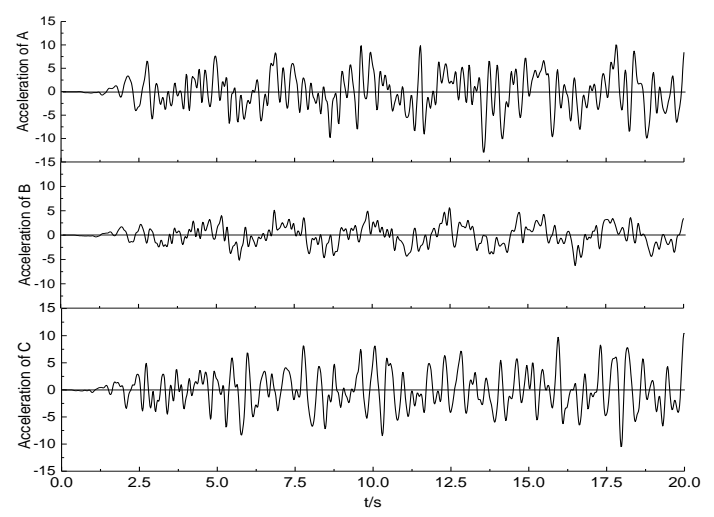

Figure 8 The acceleration response at area III

\subsection{The acceleration response of rocks in area IV}

Compared with homogeneous soil slopes, it is enormous that the influence of the distribution of rocks at the toe of slope (area IV) on the acceleration response characteristics of slope. The acceleration response curve of toe of slope point $\mathrm{C}$ is significantly different from that of the homogeneous soil slope in amplitude magnitude and variation pattern. The acceleration of toe of slope reaches the maximum of $10.87 \mathrm{~m} / \mathrm{s}^{2}$ at $18.7 \mathrm{~s}$, which is greater than the maximum acceleration of the homogeneous soil slope, and the average of peak value of the acceleration is greater than that of the homogeneous soil slope. There is also a clear "bias" in the acceleration time-history curve. It shows that the distribution of rocks at the toe of slope (area 4) will increase the acceleration response of the toe of slope.

The acceleration response on point $\mathrm{B}$ is similar to that of the homogeneous soil slope. The maximum acceleration occurs at $14.78 \mathrm{~s}$ and the value is $8.1 \mathrm{~m} / \mathrm{s}^{2}$. It shows that the distribution of rocks at the toe of slope has little effect on the acceleration response of the midslope.

The high-frequency waves in the acceleration time histories of point A are significantly reduced, and the maximum acceleration is also less than that of the homogeneous soil slope. The maximum acceleration appears at $13.72 \mathrm{~s}$ and the value is $9.94 \mathrm{~m} / \mathrm{s}^{2}$, indicating that the distribution of rocks at the toe of slope has a certain reducing effect on the high frequency seismic response on the slope top. Comparing to the acceleration response amplitudes at the slope top and the toe of slope, it shows that the acceleration response amplitude of the slope top is smaller than the acceleration response of the toe of slope, when the rocks are accumulated at the toe of slope (area IV). 


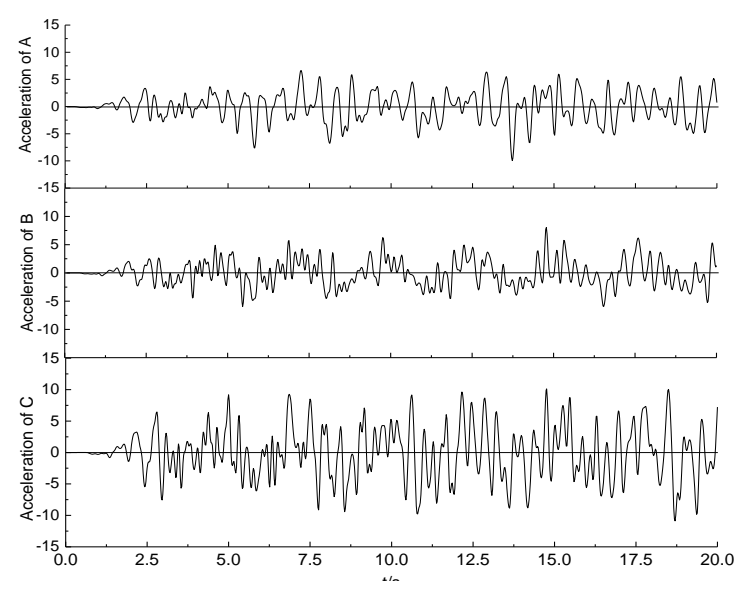

Figure 9. The acceleration response at area IV

\section{Conclusion}

By analyzing the acceleration responses of soil-rock slopes under the action of EI-centro seismic waves, conclusions are as follow:

1) Under seismic loading, the free surface of the slope makes its acceleration response characteristic different from the homogeneous soil slope, existing a high-frequency filter effect, seismic acceleration amplification effect, and the acceleration response related to the distributions of measures on the slope.

2) The distributions of rocks have great influence on the acceleration response of the slope surface. The distribution of rocks in the slope has certain reduction effect on the horizontal acceleration response at the toe of slope and the mid-slope, but has little effect on top of slope. When the rocks distribute at the slope top, the maximum value of the horizontal acceleration at the slope top and the mid-slope will decrease, and the maximum value of the acceleration at the toe of slope will increase. When the rocks distribute on the mid-slope, the maximum value of the horizontal acceleration at the slope top and the toe of slope will increase, and the maximum value of the acceleration of the mid-slope will decrease. When the rocks distribute at the toe of slope, the maximum acceleration of the toe of slope slightly will increase, the influence of the acceleration in the mid-slope is small, and the maximum value of the acceleration at the slope top will decrease.

3) There are different degrees of "bias" in the acceleration response curves of different rubble distribution locations and different slope surface measuring points. This bias is more obvious in the midslope and slope top. The characteristics of "bias" of horizontal acceleration will have an impact on the stability analysis of slopes, which should be paid attention to.

\section{Acknowledge}

This work was financially supported by Jiangsu Provincial Academy of Natural Science Research Project
Funding (17KJB560003) and National Science Fund subsidized project (51579119)

\section{References}

1. $\mathrm{Xu}$ Wen-jie,Hu Rui-lin, Conception,classification and significations of soil-rock mixture[J], Hydrogeology \& Engineering Geology, 2009, 36(4):50-56.

2. $\mathrm{Xu}$ Chong ,Xu Xi-wei,YU Gui-hua,Study on the characteristics,meichanism, and spatial distribution of Yushu earthquake triggered landslides[J], Seismology and Geology, 2012, 34(1):47-62

3. Guo Yayong,Ge Yonggang etc, Chen Xingchang,Basic caracteristics and failure mechanism of the ganjiazhai landslide triggered by the ludian earthquake,Yunnan[J], Mountain Research,2016, 34(5):530-536.

4. Chen Ling ling, Chen Min zhong,Qian Sheng guao,Stability analysis of high-steep rocky slope under earthquake loads[J], Journal of Yangtze River Scientific Research Institute, 2004, 21(1):33-35.

5. Zhang Zhuoyuan,Fundamentals of The Engineering Geology Analses[M], Geological publishing house, 2009

6. Wang Xiuying,Study of fast evaluation technique of earthquake induced landslides and their effects on earthquake emergency resuce[J], Recent Developments in World Seismology, 2011, 29(7):2159-2159

7. Ren Ziming,Dynamic response and stability of slope under seismc excitations[D], Southwest Jiaotong University,2007

8. Liu Hanxiang,Xu Qiang,Fan Xuanmei, Influence of ground motion intensity on dynamic response laws of slope accelerations[J], Earthquake Engineering and Engineering Dynamics, 2012, 33(2):41-47

9. Zheng Yingren,Ye Hailin,Huang Runqiu etc, Study on the Stability Analysis of Earthquake Slope[C], Independent Innovation and Sustainable Growth Annual Meeting of China Association for Science and Technology,2009

10. Men Ni,Sun Youwei,Bo Jingshan etc, Study on dynamic response and influence factors of slope under earthquake[J],World Earthquake Engneering, 2017(3):110-120.

11. Cen Lingling,Chen Minzhong,Qian Shengguo, Stability analysis of high-steep rocky slope

12. under earthquake loads[J], Journal of Yangtze River Scientific Research Institute, 2004, 21(1):33-35.

13. Liu Hanlong,Fei Kang,Gao Yufeng, Time history analysis method of slope seismic stability[J],Rock and Soil Mechanics, 2003, 24(4):553-556

14. Xie Hongqiang,He Hongda,Fu Wenxi,Study on dynamic pesponse and stability of slope with compound accumulationnody under strong ground 
motion[J],Sciencepaper Online, 2010, 05(7):569574.

15. Ma Fangfang,Study on the seismic stability of slope on time history analysis method and finite slement method[D],Dalian Univesity of Technology,2005 